\title{
Design and Implementation of a Driving Circuit for an Optical System Transmitter and Receiver
}

\author{
Kanat M. Abdul Hussain \\ Department of Engineering, University of Technology, Baghdad, Iraq \\ kanataltimimi@yahoo.com, +9647810845790
}

\begin{abstract}
For the laser driver circuit driving a laser diode to generate an optical output corresponding to an input data, thus, controlling the optical output based on an output current from a photodiode that detects the optical output. The output current is converted into a voltage signal whose amplitude is detected after filtering the voltage signal by a low-pass filter. Therefore, a reference signal corresponding to the input data is generated and its amplitude is detected. We have a modulator for generating a modulation current, it regulates the amplitude of the modulation current, according to the difference between the two detected amplitudes.
\end{abstract}

Key words: Corresponding, optical output, amplitude, voltage signal, detected, modulation

\section{INTRODUCTION}

A typical set up transmission system includes a transmitter, the receiver and a data channel as illustrated in Fig. 1. The signal at the transmitter will produce a suitable mode signal which transmits at the data channel, whereby the the signal passes through the data channel and sends it to the receiver (Kumar, 2008). The data channel can be divided into two types, these are the unguided channel and the guided channel. The common example of unguided channel is by using the wave motion of atmosphere or air to transmit signal and an example of a guided channel is the optical fiber (Raj, 2015). In Fig. 2 illustrates the block diagram of the optical fibers

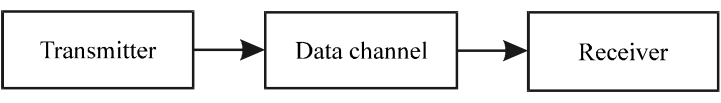

Fig. 1: The basic communication system

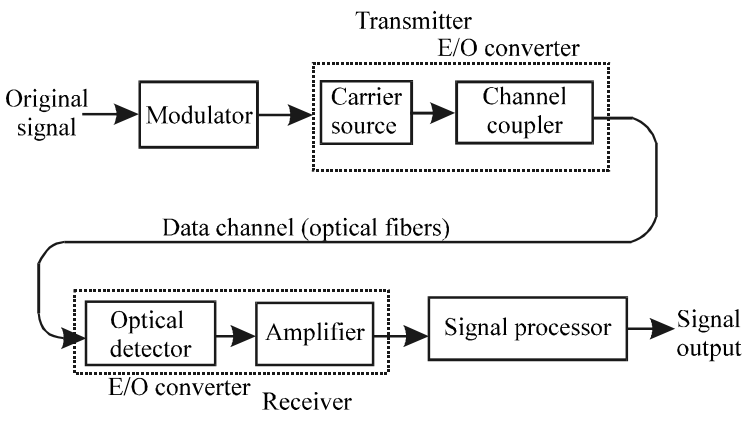

Fig. 2: The block diagram of optical fibers transmission system transmission system whereby the optical fibers transmitter includes a modulator, a carrier source and a channel coupler, thus, the optical fibers receiver includes an optical detector, amplifier and the signal processor, therefore, the data channel uses optical fibers as the guided line (Kharraz and Forsyth, 2013).

Significant of the statement: In accordance to the highlighted significance of the FSO communications and thus realizing it as an effective means in the modern day communications systems, it is therefore, worthy finding out the suitability of the system configuration and the parameters that optimized the system performance and reduces the setbacks of the atmospheric environment. The study undertaken in the research paper entails, giving out a general guideline and the performance characteristics in the study design, the simulation, the implementation, evaluation and the comparison of the proposed advanced link, through considering the performance of the current innovations in the radio over FSO system.

\section{MATERIALS AND METHODS}

Original signal: The original signal is possible with a sound wave, image or current signal (Chen et al., 2012). Therefore, in an optical fiber communication system, irrespective to the conditions in place, the data must be converted to an electric signal before it's transmitted, thereafter, a transducer is used to convert the non-electric signal to electric signal (Majumdar, 2014). This is mostly when the microphone converts the sound wave to current 
signal while the video camera converts the image to current signal as Mccormick (2014) puts it. Illustrations in Fig. 2 elaborates on how the transmitter receives the original signal, the signal then passes through a modulator which converts it to a suitable mode, thus, the signal is added to the carrier signal which is produced by the carrier source (Strobel, 2016).

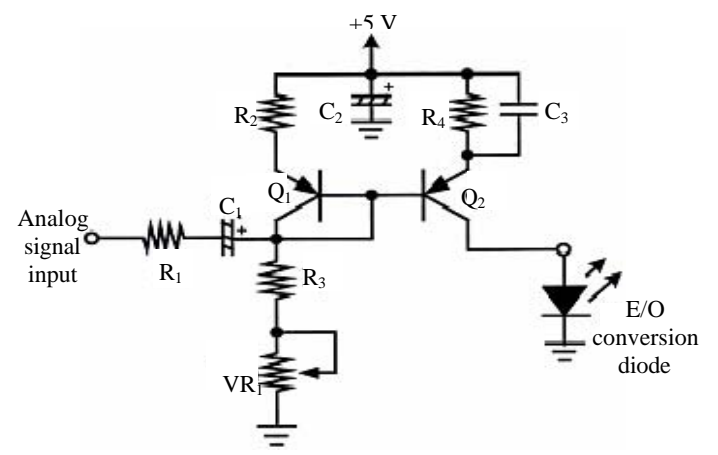

Fig. 3: The circuit diagram of analog signal modulated transmitter

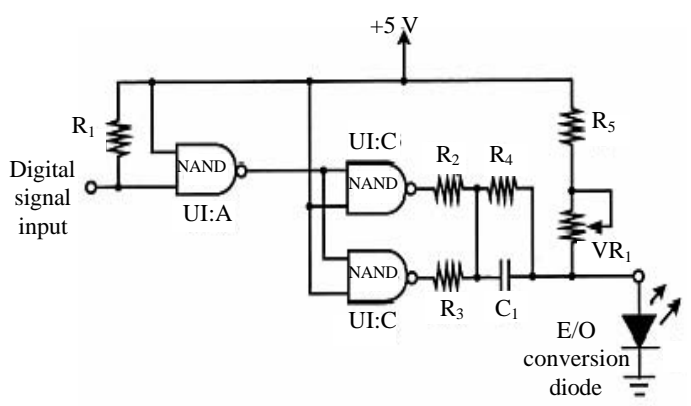

Fig. 4: The circuit diagram of digital signal modulated transmitter

(a)

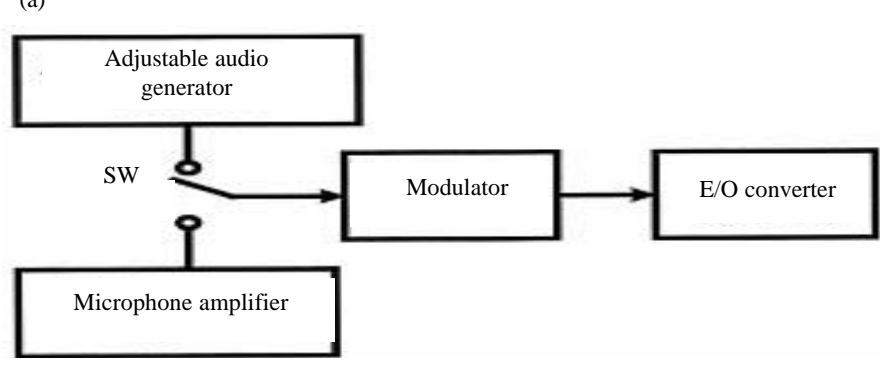

(b)

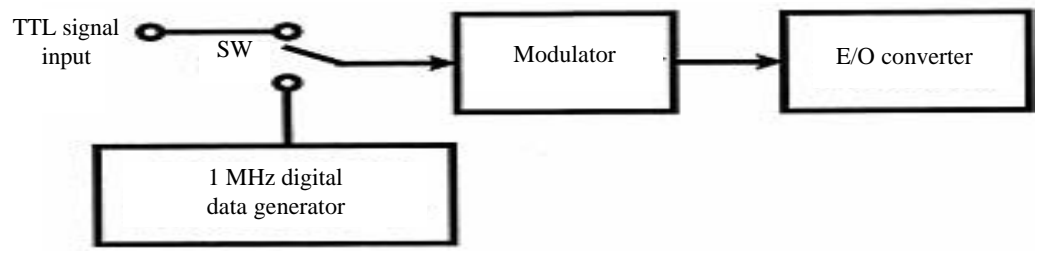

Fig. 5: a, b) The block diagram of the optical fibers communication transmitter 
sent to Electric-to-Optical $(\mathrm{E} / \mathrm{O})$ conversion interface. Whereby, we have two common types of $\mathrm{E} / \mathrm{O}$ conversion interfaces which are Light Emitting Diode (LED) and the Laser Diode (LD) (Tse and Viswanath, 2005).

The design and implementation of transmitter: The signal of the light can be used to transmit digital and analogue signals (Torres, 2013). The digital signal is the basic TTL signal with a voltage level that starts from $3.3 \mathrm{~V}$ to the voltage level of CMOS. Analogue signal includes the sine wave, the triangle wave and the square wave, therefore, the different types of modulations in the communication systems and the entire basic digital or the analogue signal can be transmitted by using the optical fibers or the free space (Oyerinde, 2011). In Fig. 5, we have the block diagram of the optical fibers communication transmitter. The diagram is divided into two main parts which are the block diagram of analogue transmitter as illustrated in Fig. 5a and the block diagram of digital transmitter as illustrated in Fig. 5b.

\section{RESULTS AND DISCUSSION}

Optical-to-Electric (O/E) conversion interface: The primary objective of the optical-to-electric converter in Fig. 6 is to convert the light wave to a current signal (Andrews and Phillips, 2005). The output current of the photo detector is proportional to the power of the incident wave and the variation of the light power contains the data signal, therefore, the output current of the photo detector also contains this data signal (Zhu and Khan, 2002). Generally, the common photo detector is the photodiode which converts the light power to current signal. Basically, the photo detector can be classified into two types which entails direct conversion of photon to electron converter and heat parameter type converter (Skraparlis et al., 2010).

The photodiode belongs to the first type which can absorb the energy of photon and produce electron, hole, and then produce the current. To add on, the bolometer belongs to the second type, whereby its value of resistance can be varied by absorbing the energy of light. In the application of high speed of rate, the first type is more suitable (Bekkali et al., 2009).

Signal processor: The main objective of the signal processor is to amplify and filter the signal from the $\mathrm{O} / \mathrm{E}$ converter, the signal processors are analog received signal processor and digital received signal processor. Figure 7 is the circuit diagram of the analogue received signal processor. In the figure U1 is an $\mu \mathrm{A} 733 \mathrm{IC}$ which is manufactured by TI company. Figure 8 is the pins diagram of $\mu \mathrm{A} 733$. This $\mathrm{IC}$ is a video frequency differential amplifier whereby its frequency band can reach $100 \mathrm{MHz}$ and the input impedance is $250 \mathrm{k} \Omega$. In the circuit, the main

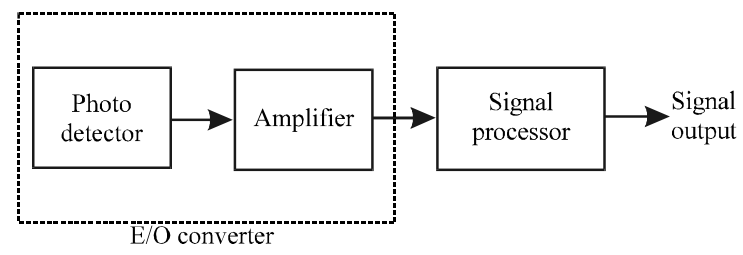

Fig. 6: The block diagram of optical fibers receiver

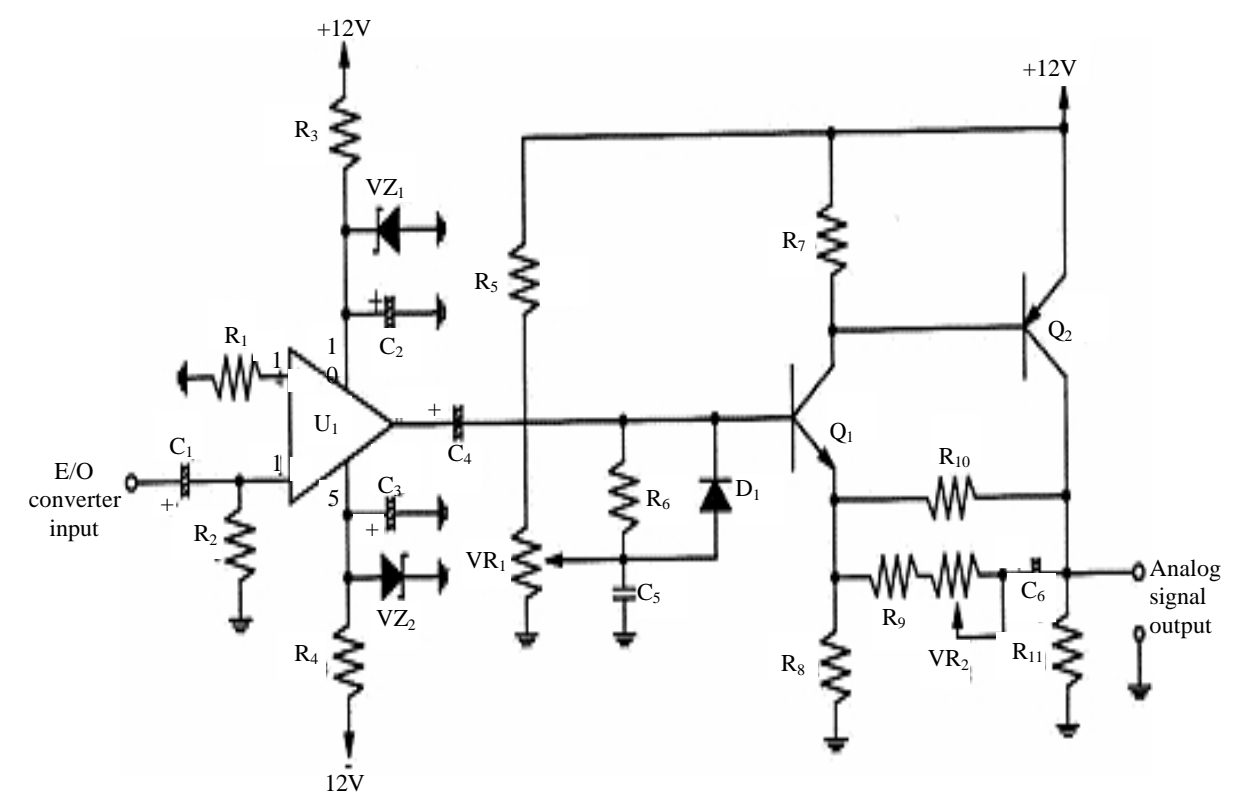

Fig. 7: The circuit diagram of analog received signal processor 
function of $\mu \mathrm{A} 733$ is for amplification of small signal. Since, the O/E converter converts the signal, the amplitude of analogue signal is very small, thus, it needs a higher gain amplifier to amplify the analogue signal and if the gain control is not connected, then there are three selections of the gain of the differential amplifier which are 10,100 and 400 times. Therefore, the selection depends on the characters at the numbers of IC. In Fig. 7, it's seen that the input signal is located at pin 1 and the output signal is located at pin 8. VZ1 and VZ2 are the voltage regulated components. Since, the operation voltage of $\mu \mathrm{A} 733$ is about $\pm 8 \mathrm{~V}$, thus, the power supply of the whole circuit is $\pm 12 \mathrm{~V}$. This means that, we need the voltage regulated components, so that, the amplifier can operate at a suitable voltage (Kreugle, 1998). Q1 and Q2 comprise of a Darlington pair series amplifier which amplifies the output signal of $\mu \mathrm{A} 733$. R9, VR2 and $\mathrm{C} 6$ which are the negative feedback channel of AC signal. R8, R10 and $\mathrm{R} 11$ are the DC bias components and R10 is also a negative feedback component. The total gain of the

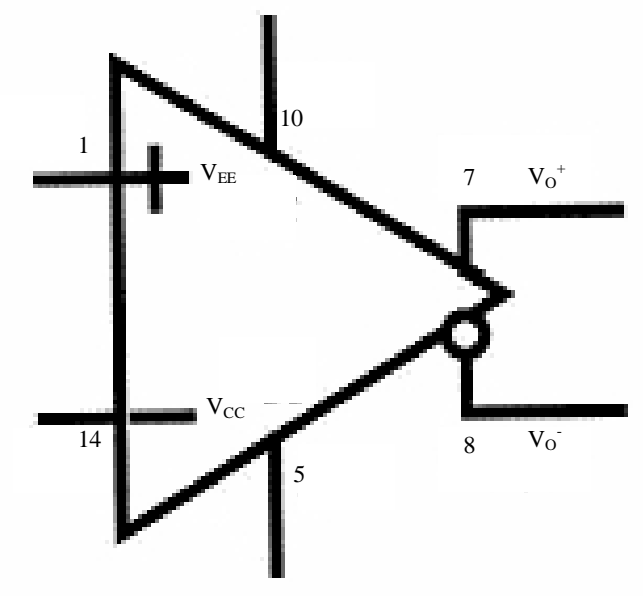

Fig. 8: The pins diagram of $\mu \mathrm{A} 733$ circuit can, therefore be varied by adjusting VR1 and VR2, so that, the output amplitude is maximum without distortion.

In Fig. 8 shows the circuit diagram of digital received signal processor. As the signal received is possibly very small or very large, it leads to the utilization of the MC101 16 IC from the Motorola Company. MC10116 is the differential amplifier which promptly process the digital received signal and also is suitable to use for long distance transmission (Kazaura et al., 2007). In Fig. 9, there are three differential amplifiers that ensures the signal can be amplified and the noise can be removed effectively. Q1 and Q2 comprise a differential amplifier, whereby it's objective is to amplify the signal of MCI0116. Since, the output of MC10116 is unable to reach the level of TTL, this makes room for transistors in achieving it for example when we design the operation mode of Q1 and Q2 as a switch and the output amplitude can be varied by adjusting VR14.

The larger the value of the resistor, the larger the output amplitude, however, the maximum need not exceed the TTL level.

The design and implementation of receiver: In Fig. 10, it illustrates the block diagram of the optical fibers communication receiver that can be divided into two primary parts. The first part is the block diagram of the analogue signal receiver as illustrated in Fig. 10a and the other part is the block diagram of the digital signal receiver as illustrated in Fig $10 \mathrm{~b}$.

In Fig. 10a, the analogue signal receiver will not only recover the signal from the transmitter but will also amplify the analogue signal by using the amplifier (Mahdy and Deogun, 2004). In Fig. 10b of the digital signal receiver, it can be seen that the weak square wave signal is amplified to TTL level by using the differential amplifier.

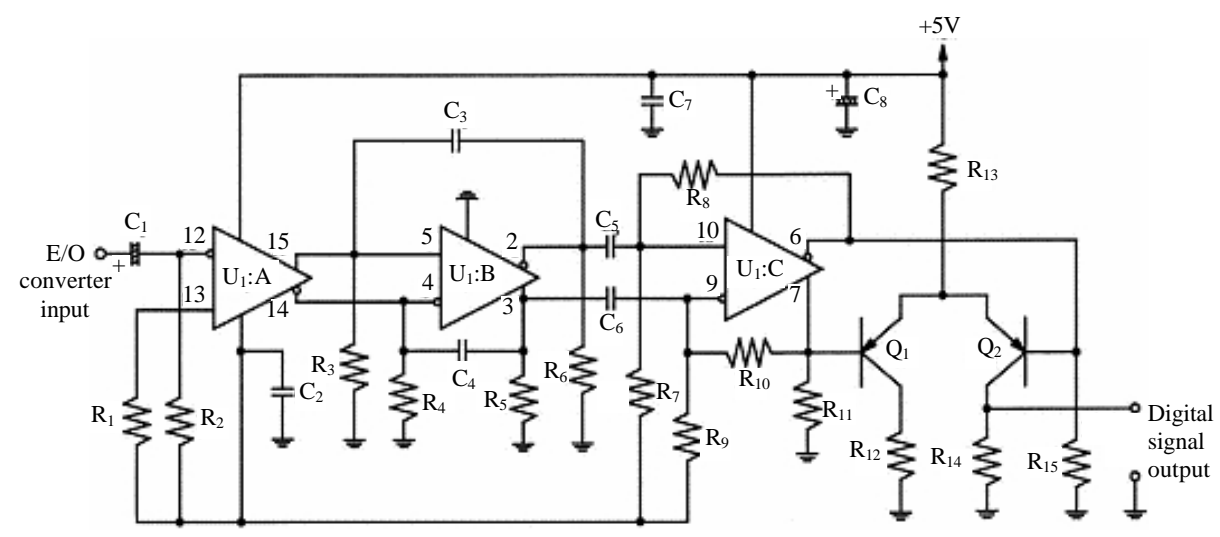

Fig. 9: The circuit diagram of digital received signal processor 


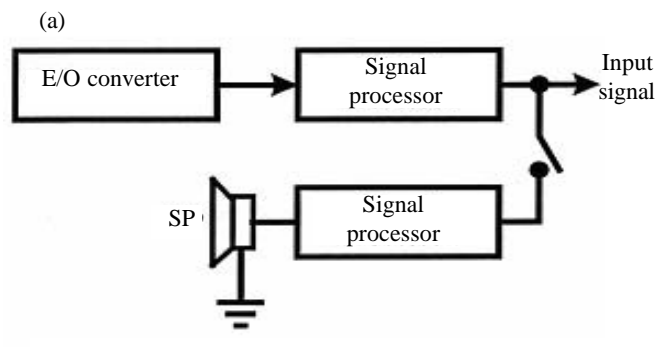

(b)

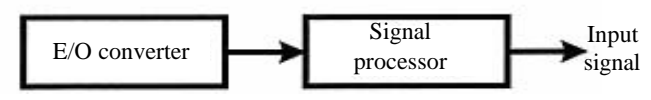

Fig. 10: a, b) The block diagram of optical fibers communication receiver

\section{CONCLUSION}

The project under study explains how to connect up and build a laser diode circuit. A laser diode is a diode which outputs a laser beam. Unlike the LED light, a laser's light output is more concentrated, meaning it has a smaller and more narrower viewing angle (Abaza et al., 2013). This means it must be directed at its source more directly in order to be picked up. The laser light is also a monochromatic, meaning laser light isn't composed of several lights combined together but one light of the same wavelength and energy (Rahman et al., 2012). The laser lights have a single spectral color and is almost the purest monochromatic light available. A laser diode circuit is built and a driver circuit for the laser diode is created. A driver circuit is a circuit which can limit appropriately the amount of current being fed into the laser diode, so that, it can function correctly (Manor and Arnon, 2003). Too much current and the laser diode will blow. Too little current and the laser diode will not have sufficient power to turn on and operate. Therefore, a driver circuit is needed to give precisely the correct range of current needed, so that, the diode will operate in build the driver circuit, a voltage source, a voltage regulator, a diode, an electrolytic capacitor and a few resistors are needed (Sangeethan et al., 2014). All these parts were explained in the previous chapters under the study.

\section{ACKNOWLEDGEMENT}

I would like to thank my entire family for the support and strength they have given me to undertake this academic research and to my supervisors Dr. Shebab Kadhim and Dr. Alaa Ali for the tireless support and encouragement during my research sessions. May God bless you all.

\section{REFERENCES}

Abaza, M.R., R., Mesleh, A. Mansour and A. Alfalou, 2013. MIMO techniques for high data rate free space optical communication system in log-normal channel. Proceedings of the International Conference on Technological Advances in Electrical, Electronics and Computer Engineering (TAEECE), May 9-11, 2013,IEEE, Konya, Turkey,ISBN: 978-1-4673-5612-1, pp: $1-5$.

Andrews, L.C. and R.L. Phillips, 2005. Laser Beam Propagation through Random Media. SPIE Press, Bellingham, Washington, USA., ISBN:9780819459480, Pages: 782 .

Bekkali, A., P.T. Dat, K. Kazaura, K. Wakamori and M. Matsumoto et al., 2009. Performance evaluation of an advanced DWDM RoFSO system for transmitting multiple RF signals. IEICE. Trans. Fundam. Electron. Commun. Comput. Sci., 92: 2697-2705.

Chen, Z., S. Yu, T. Wang, G. Wu and S. Wang et al., 2012. Channel correlation in aperture receiver diversity systems for free-space optical communication. J. Opt., 14: 125710-125710.

Kazaura, K., K. Omae, T. Suzuki, M. Matsumoto and E. Mutafungwa et al., 2007. Performance evaluation of next generation free-space optical communication system. IEICE. Trans. Electron., 90: 381-388.

Kharraz, O. and D. Forsyth, 2013. PIN and APD photodetector efficiencies in the longer wavelength range 1300-1550 nm. Optik Intl. J. Light Electron Opt., 124: 2574-2576.

Kreugle, H., 1998. CCTV Surveillance: Video Techniques and Technology. Butterworth-Heinemann, Oxford, UK., Pages: 468.

Kumar, S., 2008. Wireless and Mobile Communication. New Age International Ltd, New Delhi, India,.

Lehpamer, H., 2002. Transmission Systems Design Handbook for Wireless Networks. Artech House, Boston, London, UK., Pages: 606.

Mahdy, A. and J.S. Deogun, 2004. Wireless optical communications: A survey. Proceedings of the 2004 IEEE International Conference on Wireless Communications and Networking (IEEE Cat. No.04TH8733), March 21-25, 2004, IEEE, Atlanta, Georgia, USA., pp: 2399-2404.

Majumdar, A.K., 2014. Advanced Free Space Optics (FSO): A Systems Approach. Springer, Berlin, Germany, ISBN: 978-1-4939-1917-9, Pages: 396.

Manor, H. and S. Arnon, 2003. Performance of an optical wireless communication system as a function of wavelength. Appl. Opt., 42: 4285-4294. 
Mccormick, C., 2014. Fiber-Optic Communication 49 Success Secrets-49 Most Asked Questions on Fiber-Optic Communication-What You Need to Know. Lightning Source Printing Company, La Vergne, Tennessee, USA., ISBN:9781488525674, Pages: 118.

Oyerinde, O., 2011. Channel Estimation for Wireles Communication Systems. Lambert Academic Publishing, Riga, Latvia, ISBN:9783844397512, Pages: 228.

Rahman, M.T., S. Iqbal and M.M. Islam, 2012. Modeling and performance analysis of free space optical communication system. Proceedings of the 2012 International Conference on Informatics, Electronics and Vision (ICIEV), May 18-19, 2012, IEEE, Dhaka, Bangladesh, ISBN:978-1-4673-1153-3, pp: 211-218.

Raj, A.A.B., 2015. Free Space Optical Communication: System Design, Modeling, Characterization and Dealing with Turbulence. The Walter de Gruyter, Oldenbourg, Berlin, Germany, ISBN:9783110449952, Pages: 234.

Sangeetha, N., I.K. Pathan and V. Vinayakamurthy, 2014. Analys is of outage probability and diversity gain for MIMO free-space optical links. Intl. J. Emerging Technol. Adv. Eng., 4: 707-713.
Skraparlis, D., M. Sandell, V.K. Sakarellos, A.D. Panagopoulos and J.D. Kanellopoulos, 2010. On the effect of correlation on the performance of dual diversity receivers in lognormal fading. IEEE. Commun. Lett., 14: 1038-1040.

Strobel, O., 2016. Optical and Microwave Technologies for Telecommunication Networks. John Wiley and Sons, Hoboken, New Jersey, USA., ISBN:9781119154587, Pages: 520.

Torres, D.F., 2013. Cosmic Rays in Star-Forming Environments: Proceedings of the Second Session of the Sant Cugat Forum on Astrophysics. Springer, Berlin, Germany, ISBN:978-3-642-35409-0, Pages: 446.

Tse, D. and P. Viswanath, 2005. Fundamentals of Wireless Communication. Cambridge University Press, Cambridge, New York, ISBN-13:978-0-521-84527-4, Pages: 574.

Zhu, X. and J.M. Kahn, 2002. Free-space optical communication through atmospheric turbulence channels. IEEE Trans. Commun., 50: 1293-1300. 